\title{
Effect of Screw Diameter in Femoral Fracture Fixation Modeled by Finite Element Method
}

\author{
Muhammad Nashrullah ${ }^{1}$, Belladina Clara Shinta ${ }^{1}$, Mas Irfan P. Hidayat ${ }^{1}$, \\ Agung Purniawan ${ }^{1}$, and Yuli Setiyorini ${ }^{1}$
}

\begin{abstract}
-research in the field of biomechanics related to orthopedic implants is rapidly growing in the world, especially for fixation of fractured bone. Fixation is a surgery to locate and attach a plate pressed by screws at the fractured bone. Most of the fracture bones sited in femur. This research focuses on modeling and simulation of plate and screw made of SS316L material for the fixation of the fractured femur using finite element method (FEM). SS316L is a material with good biocompatibility, cheap, and ductile enough. Modeling and simulation done using ANSYS 15.0 software. Simulation results are stress, strain, and total deformation. It is obtained that optimum diameter of screw is $4.5 \mathrm{~mm}$ with respect to minimum value of von Mises stress and deformation.
\end{abstract}

Keywords-deformation, finite element method, screw diameter, SS316L, von Mises stress.

\section{INTRODUCTION}

Fractures occur for their loading on the bone exceed the strength of the bone itself, caused by motor vehicle accident for example. The latest data released by the World Health Organization (WHO) shows that Indonesia is the fifth case of motor vehicle accidents in the world. Data from the Indonesian National Police recorded during the year 2013 happened 93,578 cases of motor vehicle accidents. Almost $80 \%$ of the accident victims suffered fractured bones. Fractured bones can be restored by arranging th position of fractured bones so close to each other as well as to keep them not shift and stick together. The bones can heal themselves by the time. There are two methods of recovery of bone that is often applied in the field of medicine; they are external and internal fixation. External fixation is the installation of gypsum which is a strong material that is wrapped around the outside of the body where the fractured bone discovered so the position not shifted. However, installation of gypsum can limit patient movement activities. Popular method today is the internal fixation. This method is surgery to place and attach a metal plate that is reinforced with screws on around the fractured bone. This implants are temporary, after the bones recovered then the plate and screw can be taken through surgery back.

Femur or thigh bone is the longest bone of the body. The bone jointed with the acetabulum in the hip socket formation and from here extends medial to the knee and make the joints with the tibia. Her bone in the form of bone pipe and has a stem and two ends, namely the upper end, and the lower end of the femoral shaft. In the case of bone, rigidity is determined by the relative proportions of crystalline hydroxyapatite and collagen threads that make up the composite. Ultimate stress occurs when loading

${ }^{1}$ Muhammad Nashrullah, Belladina Clara Shinta, Mas Irfan P. Hidayat, Agung Purniawan, and Yuli Setiyorini are with Departement of Material and Metallurgical Engineering, Institut Teknologi Sepuluh Nopember, Surabaya, 60111, Indonesia. E-mail: nashrullah14@mhs.mat-eng.its.ac.id; bella_clara92@gmail.com; continues in the area of plastic, and eventually the material will reach the point of failure of the primary, in which the specimen is broken. The point at which a broken bone can be seen as the ultimate strain or ultimate stress $(140 \mathrm{MPa}$ of compression, $200 \mathrm{MPa}$ for tension, and $65 \mathrm{MPa}$ for shear) [1].

The yield stress is when an increase in strain is no longer directly proportional to the stress, in which the elastic deformation region ends. At the same time the material loses its ability to return to the original shape. Specimens have been transformed into the plastic areas where irreversible damage is already happening. In the case of bone, the failure occurred because microcracks in the ultrastructural disruption of hydroxyapatite and collagen threads. For cortical bone, the result of the yield stress is about $130 \mathrm{MPa}$.

Elasticity is the ability of an object to return to its original shape as soon as the external force given to it removed. An object can be said to be perfectly elastic if the force causing the deformation missing object will return to its original shape. Objects that are perfectly elastic which have limits of deformation is called elastic limit so that if it exceeds the elastic limit, the body will not return to its original shape. The difference between elastic and plastic properties are in large or small levels of deformation. Deformation of the object will cause change in shape but there is no change in volume, and objects that compression volume change will occur but not deformed.

Classical elasticity theory shows the relationship between deformation, force, strain, and stress. When a solid object is given external forces, the object is deformed and produces internal strain and stress. Deformation depends on the configuration of the geometry of objects, a large load is given, and the mechanical properties of the material of the object itself. Classical elasticity theory assumes that the material is homogeneous and isotropic, ie that the mechanical properties of the material is the same in all directions and all points.

Each material is elastic in its natural state. Therefore, if the external force acting on an object, the object will be deformed. When the object is deformed, the molecule will form a resistance to deformation. This prisoner per unit area known as stress. Stress is defined as the force needed by the body to return to its original shape. Von Mises failure theory introduced by Huber (1904) and then refined through the contribution of Von Mises and Hencky. This theory states that failure is predicted to occur in multiaxial stress state when the distortion energy per unit of volume equal to or greater than the distortion 
energy per unit volume in the event of a failure in a simple uniaxial tension test on a specimen of the same material. In general, the material shows the phenomenon of multiaxial stress, so that the criteria used to connect the elongation of multiaxial stress with uniaxial tension. Von Mises failure theory predicts that the elongation will occur if the voltage exceeds the voltage equivalent uniaxial creep. Therefore, the failure occur when the value of von Mises stress surpass the material's yield strength value.

Orthopedic implants can be defined as medical devices used to replace or provide fixation of bone, or to replace articulating surfaces of a joint. Orthopedic implants are are mainly made from biomaterials. Biomaterials have been defined as materials used in medical devices and are intended to interact with biological systems living body [2]. In the development of medical implants, a lot of consideration, among other mechanical properties (strength, endurance, and so on), function (interaction between the implant and the body), and the relationship between the implant to a specific design. First and foremost is the biomaterial must compatible with the human body. These biomaterials have to show no adverse response from the body, or the reverse, should be nontoxic and non-carcinogenic. In addition, the biomaterial must have the physical and mechanical properties sufficient to serve as a replacement or a multiplier of body tissue.

TABLE 1.

VON MISES STRESSES AND STRAINS VALUE OF ALL COMPONENTS

\begin{tabular}{|c|c|c|c|c|c|c|c|c|c|}
\hline \multirow{2}{*}{$\begin{array}{l}\text { Diameter of } \\
\text { Screw }(\mathrm{mm})\end{array}$} & \multicolumn{3}{|c|}{ Von Misses Stress Value (MPa) } & \multicolumn{3}{|c|}{ Strain Value } & \multicolumn{3}{|c|}{ Total Deformation Value (mm) } \\
\hline & Screw & Plate & Femur & Screw & Plate & Femur & Screw & Plate & Femur \\
\hline 4.5 & 126.36 & 124.87 & 45.374 & 0.0006547 & 0.00064701 & 0.0025349 & 0.56953 & 0.65009 & 1.8870 \\
\hline 4.0 & 218.86 & 167.66 & 93.997 & 0.0012360 & 0.00094318 & 0.0052512 & 0.47578 & 0.54901 & 1.6844 \\
\hline 3.5 & 243.38 & 176.68 & 65.461 & 0.0014683 & 0.00102950 & 0.0038769 & 0.61232 & 0.70827 & 2.0319 \\
\hline 3.0 & 287.41 & 212.68 & 84.242 & 0.0014892 & 0.00110190 & 0.0047602 & 0.64825 & 0.75700 & 2.1676 \\
\hline 2.7 & 359.22 & 197.48 & 290.63 & 0.0024188 & 0.00112770 & 0.0162360 & 0.66084 & 0.77330 & 2.2075 \\
\hline
\end{tabular}

Many types of biomaterials are used, including metals, alloys, ceramics, polymers, composites, and glass. Metal has a many scopes in application. The elastic modulus and yield strength combined with metal ductility makes this material is sustainable to load without causing deformation. The common metal used is titanium alloy, stainless steel, and $\mathrm{Co}-\mathrm{Cr}$ alloys. Its application in the field of medicine is on the plate and bone screws. The use of plates and screws first documented in the 1880s and 1890s. Materials commonly used are stainless steel 316L. The advantage of this material is cheap price and easy to produce, tolerance major surgery, and a lot of in-depth research on this specimen.

Use of plates, screws, and wires was first documented in the 1880 s and 1890s. Early surgical fixation initially was complicated by many obstacles, such as infection, poorly conceived implants and techniques, metal allergy, and a limited understanding of the biology and mechanics of fracture healing. During the 1950s, Danis and Muller began to define the principles and techniques of internal fixation. Over the past 40 years, advancements in biological and mechanical science have led to contemporary fixation theories and techniques. In the development of medical implants, many considerations of mechanical properties (strength, endurance), functionality (interaction between the implant and the body) and implant specific design. Of course, the properties mentioned above are specific to each implant type and have to be considered case-by-case [3]. Some of the risks that occur when using metal implants in the body among other related aspects of corrosion, implant failure due to fatigue, elongation (creep) of metals, metal stress, and more. Metal implant failure due to fatigue occurs due to repeated loading under their ultimate strength. Endurance limit is the amount of voltage applied to the material in which the materiality will not fail even after several million cycles of loading.

Orthopaedic screws are used as the primary mode of fixation for applications such as fracture plates [4], intramedullary rods [5], spinal devices [6] and suture anchors[7]. The ability of devices to perform as designed is dictated by pullout strength of the screws anchoring them. Pullout is a commonly reported clinical problem [8]. The failure mechanisms leading to pullout have not previously been uncovered. The main goals of any orthopedic fixation technique are to maintain anatomic alignment, provide stability of the fracture to facilitate healing, and increase load bearing to facilitate rehabilitation. Internal fixation is commonly a fracture spanning plate with a combination of screws through the plate. The two main drawbacks of internal fixations are disruption of blood supply and shielding the underlying bone and fracture gap from mechanical stresses. To overcome vascularisation issues and surgical exposure, orthopedic hardware is installed with utmost care to protect soft tissues, and minimize soft tissue stripping in order to lessen devascularisation of bone [9].

The study aimed to predict the number and position of screws needed to accelerate fracture healing process but still acceptable in terms of biomechanics was done by Nasr et al [9]. Modeling and biomechanical analysis performed using Abaqus software. Plates and screws are 
made of SS316L material. This study uses a plate with the number of 8 holes units with screw diameter is $4.5 \mathrm{~mm}$. According to Nasr, the optimal number of screws as many as 4 pieces. Based on the von Mises stress distribution spectrum, a large maximum stress on the screw is equal to $473 \mathrm{MPa}$, while the plate is equal to $226 \mathrm{MPa}$ which is in the region of the hole. Screw receives the stress distribution from plate and bone. The stress on the screws is $30 \%$ more than at the plate because its function to lock plate and bone together.

When a human bone fracture occurs, various types of internal fixation devices like bone plates are applied to the fracture site to promote bone structure stabilization. As a rule, such bone plates are used to fix long-bone fractures with several fastening screws and are usually made of non-corrosive metals such as stainless steel and titanium alloys. Therefore, a pre-estimation of the stresses which may subject to those plates gains importance. Finite Element (FE) analysis has recently been used by numerous researchers to predict the structural behavior of the bone with plates under a considered load [10].

Finite element method is a numerical method in which the idea of this method is divide the structure into smaller parts (elements), each with simple geometries with reason easier to analyze than the original structure. The elements are connected to one another in points called nodes. Thus, in a series of many simple calculation, a solution can be found as an approach to form complex structures, depending on the class or level of simplification in finite element models.

Research related internal fixation on the femur was also conducted by Lee et al [11]. The difference with Nasr, Lee uses a variation of 12 holes on the plate. Imposition of a given set the direction of the $\mathrm{x}, \mathrm{y}$, and $\mathrm{z}$. For muscle force in the $\mathrm{x}$-axis is $320 \mathrm{~N}$, on the $\mathrm{y}$-axis is $170 \mathrm{~N}$, and the $\mathrm{z}$ axis is $2,850 \mathrm{~N}$. At region of the hip joint on the $\mathrm{x}$ axis is $-300 \mathrm{~N}$, on the $\mathrm{y}$-axis at $0 \mathrm{~N}$, and the axis $\mathrm{z}$ is $-1,200 \mathrm{~N}$. Plates and screws are made of SS316L material with a screw diameter of $3.5 \mathrm{~mm}$. The results show that the optimum number and position screw when put six screws. Numerical analysis was performed using ANSYS software. On the screw arrangement provide uniform stress distribution and the minimum to all parts of the bone, screws, and plates. Based on the numerical calculation results can be given the value of the maximum load that can be accepted implant is equal to 3,608 MPa.

The study was conducted Sepehri et al. [12] in 2013 to do research related to the composition of the distribution of the screw in the plate against the biomechanical effects on the tibia. Sepehri using a plate with a hole total of 11 pieces, where the fracture is located on the 6th hole. Preparation of optimal screw obtained from this study is that if a screw inserted in the holes 1, 2, 5, 7, 10, and 11 . At the position of the preparation of a response when the minimum load of $500 \mathrm{~N}$ applied.

Several studies provide the different technique, materials, and combination of plate, screw, and bone. But generally the whole the research provides the same solutions related to the preparation of the position of the screw, which is placed on the end plate and the area around the fracture. Screw on end plate serves to lock and tighten the plate on the bone so that the plate does not move. While the screw is located in the area of the fracture serves to maintain the relative position of the bone fragments. By positioning the area of fracture in place without shifting, then the recovery can get faster. Of the many methods of preparation and screw position would require further research related to the same thing to validate research results that have been there.

Based on this, it is necessary to study specifically related to optimal screw diameter, because a similar study has not been developing yet. The reasons for selecting these variables because of the change of the screw diameter can affect the biomechanical strength, such as stress distribution and deformation in the screw, plate, and a femur that ultimately affect healing time.

\section{METHOD}

The components of this study were femur, plate, and screw (see Figure 1). An ideal three-dimensional finite element model of human femur was developed using Auto Cad 18.1. The fracture located at the center of cylindrical bone with no gap to represent the a transverse fracture in femoral shaft. The CAD file then imported to ANSYS 15.0. The cylindrical bone was modeled as diameter of 30 $\mathrm{mm}$ and length of $300 \mathrm{~mm}$. The femur designed as an isotropic material with Young's Modulus of 17,900 MPa, Poisson's Ratio of 0.4, Shear Modulus of 63,929 MPa and Bulk Modulus of 28,833 MPa.

To model the internal fixation, a $150 \mathrm{~mm}$ slotted plate was created with 8 holes using ANSYS 15.0. Each holes has oval shaped which one the side has slotted. Meanwhile, the screw was modeled using ANSYS 15.0 is cortical bone screw based on Patel, et al [13]. The screw is modeled based the commercial screw in 5 types diameter. They are $2.7 \mathrm{~mm}, 3.0 \mathrm{~mm}, 3.5 \mathrm{~mm}, 4.0 \mathrm{~mm}$, and $4.5 \mathrm{~mm}$. The length of all variants were $30 \mathrm{~mm}$ from the head ( $25 \mathrm{~mm}$ for the thread length). Plate and screw were designed as an isotropic SS316L material, with Young's Modulus of 193,000 MPa, Poisson's Ratio of 0.3, Shear Modulus of 74,231 MPa, and Bulk Modulus of 160,830 $\mathrm{MPa}$.

Bone plates are typically used to achieve compression. The strength of a plate is defined by $\mathrm{S}=\mathrm{BH}^{3}$ ( $\mathrm{B}=$ width, $\mathrm{H}$ $=$ height). Plate failure is usually due to fatigue and is more likely if there is a gap between bone and the plate. Plates are provided in various sizes and shapes for different bones and locations. The screw holes in a dynamic compression plate are shaped with an angle of inclination on one side away from the center of the plate. When tightened, the screw head slides down the inclination, causing movement of the bone fragment relative to the plate, are resulting in compression at fracture sites [3].

Screws are complex tools with a four part constructions: head, shaft, thread and tip. The head serves as the counterforce against which compression generated by the screw acts on the bone. The shaft or shank is the smooth portion of the screw between head and threaded region. The tread is defined by its root (or core) diameter, its thread (or outside) diameter, its pitch (or distance between adjacent threads), and its lead (or distance it advances into the bone with each complete turn). The root area determines the resistance of the screw to pull-out forces and relates to the area of the bone at the thread interface and the root area of the tapped thread. Screws break 
because of two reasons: excess of torque during insertion or by bending. After implantation, bending of the screw occurs secondary to a loose plate or bone loss due to delayed or non-union. The bending and shear strength is mainly determined by core diameter of the screw [9].

The plate and screw internal fixation technique is widely used for the treatment of fractured long bones. In this treatment, the healing of bone fractures can be assisted by screwing a rigid plate across the fracture site. The internal fixation hardware provides excellent reduction in the number of bone fragments and has the necessary strength to stabilise and support the fracture [14]. The roles of the bone-plate and screws are to hold the fractured bone segments in position without allowing tensile stress at the fractured surface and to provide a critical amount of compressive stress at the fracture interface in order to accelerate bone healing [15]. The plate and screw internal fixation technique has been improved with the presence of new biocompatible rigid materials such as stainless steel. However, bone osteoporosis is often found under these rigid plates, and bone refracture may occur after the plate is removed.

All of the components were grouped as an assembly (see Figure 2). All 8 holes in plates were inserted with screws. The screw inserted in the slotted area of the plate. The connection between plate and femur were frictionless, plate and screws were frictionless, and screws to femur were bonded. Screws were set self-taped (making hole inside femur by itself). Plate attaches femur and locked by screws. Meshing were set in finest mesh. Meshing is the process of modeling of structures/objects by dividing them into small elements (finite element) connected by dots called nodal (nodes) used by these elements and as the boundaries of the structure/object.

The assembly of all components was static structure with assumption the human stand up straight with load of $550 \mathrm{~N}$ directly on negative y-axis (see Figure 3). For $\mathrm{x}$ axis and $\mathrm{z}$-axis the load is $0 \mathrm{~N}$ each other. The load is given in hip joint area, in the upper femur that connected to pelvis. We assumed the load based on average of Indonesian human body weight [16]. Simulation results are stress, strain, and total deformation. If $\sigma$ is normal stress, $\tau$ is shear stress, and $\mathrm{F}$ is force (load), then the stress must satisfy the following differential equation of equilibrium :

$$
\begin{aligned}
& \partial \sigma_{\mathrm{x}} / \partial \mathrm{x}+\partial \tau_{\mathrm{xy}} / \partial \mathrm{y}+\partial \tau_{\mathrm{xz}} / \partial \mathrm{z}+\mathrm{F}_{\mathrm{x}}=0 \\
& \partial \tau_{\mathrm{yx}} / \partial \mathrm{x}+\partial \sigma_{\mathrm{y}} / \partial \mathrm{y}+\partial \tau_{\mathrm{yz}} / \partial \mathrm{z}+\mathrm{F}_{\mathrm{y}}=0 \\
& \partial \tau_{\mathrm{zx}} / \partial \mathrm{x}+\partial \tau_{\mathrm{zy}} / \partial \mathrm{y}+\partial \sigma_{\mathrm{z}} / \partial \mathrm{z}+\mathrm{F}_{\mathrm{z}}=0
\end{aligned}
$$

\section{RESULTS AND DISCUSSION}

Modeling and simulation of assembly consisting of a plate, screw, and femur was performed in accordance with the method. The type of screw used is cortical bone screw, because in accordance with the conditions of the femur which is mostly composed of hard cortical bone. Plate mounted on the surface of the femur by attaching the screw. Screw lock by punching holes in the femur by itself and not by punching holes in the femur first, then screw inserted in the hole. The condition of the femur plate is frictionless, the condition of the plate with a screw is frictionless, and the condition of the femoral screw is bonded. The output of the simulation of the overall assembly is in the form of the value of von Mises stress, strain and deformation that accompanied the distribution of spreading through the color spectrum image.

The objective of the present study was to explore the effect of screw diameter on the fracture healing process in a plated transverse femoral fracture. The stress analyses showed that the screws and plate experienced a higher magnitude of stress compared to the bone. Furthermore, the screws experienced a higher magnitude of stress compared to the plate which supports the clinical observation that screw breakages are more common than plate failures. This was likely because the screws were in direct contact with the bone and transferred the load directly from the bone to the plate. The basis of bone-plate fixation relies on the compression of the plate to the bone by the induced tensile stresses in the screw [17]. A higher screw tension is desirable because a higher frictional force must be overcome in order for loosening to occur, and it will also likely result in an increased transfer of mechanical stimuli to bone, which is reduce shielding.

Table 1. below showed the value of maximum von Mises stress, strain, and total deformation of all components with the screw's diameter variant. From the data stress, we know that femur has the lowest value than other screw and plate. But it has the biggest total deformation value. For stress and strain value, screw is in the middle of plate and femur, but it has the lowest total deformation value. From the data below we obtained when the screw diameter decrease, then the stress on screw itself, plate, and femur are increase. It also applies to strain and total deformation in all components. Based on the table is also known that the stress and strain is directly proportional. If the stress higher, then strain will also rise, and vice versa. But inversely when the stress is high, then the value of deformation decreases.

Meanwhile, SS316L had a yield strength value about $170 \mathrm{MPa}$. Clinically, the use of metal materials, especially stainless steel can be found in many applications. Use of SS316L often found as implants in the human body, while SS304 widely applied outside the body. Biomaterials research using more done because SS316L. SS316L properties that are more resistant to corrosion, especially corrosion of the blood so it does not poison the system, tissues, and organs in the human body. Based on Table 1. It can be concluded that the optimum screw diameter was $4.5 \mathrm{~mm}$ because it has the minimum von Mises stress, strain, and deformation value. Furthermore, the value of its stress below the yield strength value. It indicates the screw's not deformed. If the stress value surpasses the yield strength, it can lead plastic deformation. 


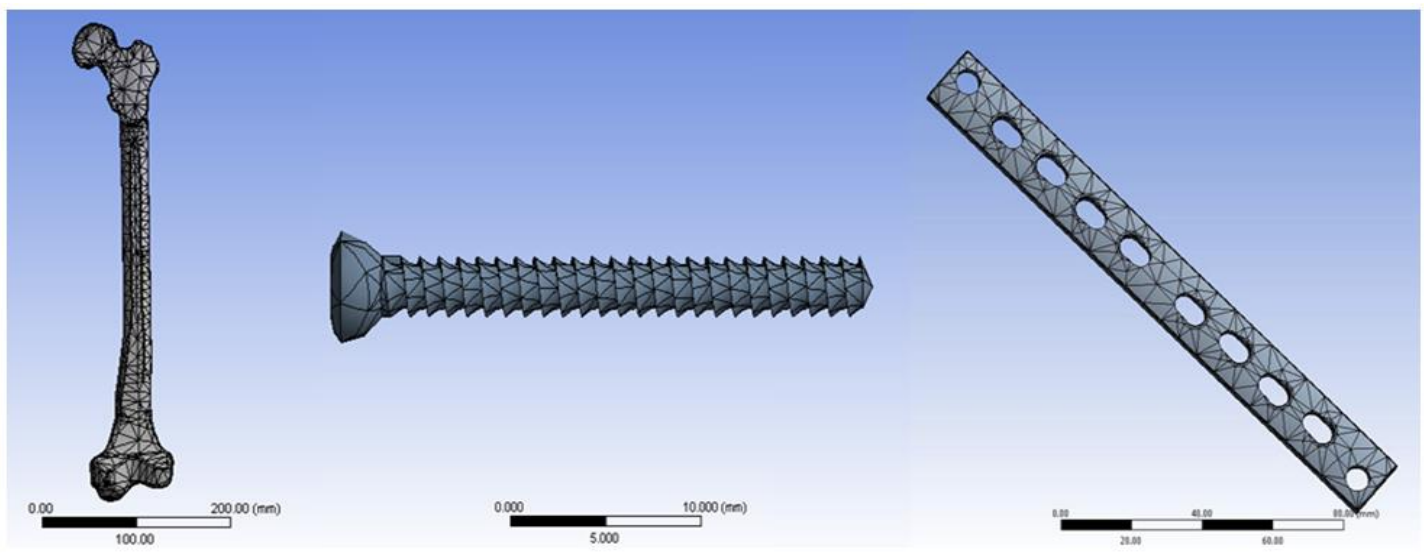

Figure 1. The finite element model of (a) femur, (b) screw, and (c) plate

Large maximum von Mises stress in the optimum screw design that is amounting to $126.36 \mathrm{MPa}$ with deformation of $0.56953 \mathrm{~mm}$ for material SS316L. Nasr, et al. in 2013 also conducted similar research using the same material and deduce the value of the maximum von Mises stress in the screw design is best of $473.25 \mathrm{MPa}$. The results obtained in this study are almost four times smaller than the result obtained by Nasr, et al. While the combination of the same design, the deformation of the largest assembly contained in the thigh bone that is equal to 1.887 $\mathrm{mm}$. This value is much greater than the deformation of the screw itself $(0.56953 \mathrm{~mm})$. This deformation has also been tested by Lee, et al. in 2012. By using synthetic conditioned femur thigh bone resembles the original, tensile test performed by placing a plate and screw in the femur. Deformation is recorded from the test results is equal to $5.63 \mathrm{~mm}$, greater than the simulation results obtained in this study.

Von Mises stress distribution of all components showed in Figure 4. The picture showed the spectrum of color that represents the stress distribution. There are shades of dark blue to red region. Dark blue region shows the minimum value of von Mises stress and strain, and vice versa ie red areas have maximum value. Figure 4a. showed the stress distribution of assembly. We noted that the maximum value of all assembly was $126.36 \mathrm{MPa}$. Then the assembly can be separated into its components, such as femur (Figure 4b.), plate (Figure 4c.), and screw (Figure 4d.) For femur, maximum stress is found inside the bottom hole far from the given load. The same thing is found in the plate. Maximum stress also found in screw inserted in the same hole which femur and plate have maximum stress value located. Based on assembly, it is known that the maximum stress located in screw. A region with a large von Mises stress is on the screw located in thread which closest to the screw head, with distribution color of orange to red. This thread located at the border area between plate and femur. There is a stress concentration in this area because screw received weightbearing from plate and bone. Meanwhile, the threaded part of screw which completely submerged in the femur have blue color indicates smaller stress value.

As the strain, there is also strain. Figure 5. shows strain distribution. The picture showed the spectrum of color that represents the strain distribution in assembly (Figure 5a.), femur (Figure 5b.), plate (Figure 5c.) and screw (Figure 5d.). These spectrum distributions were same as the spectrum distribution in von Mises stress. There are shades of dark blue to red region. Dark blue region shows the minimum value of von Mises stress and strain, and vice versa ie red areas have maximum value. The color distribution change follows the visible light spectrum. The strain pattern is similar to stress pattern. Maximum strain in assembly is found inside the bottom hole far from the given load. The same thing is found in the femur, plate, and screw. Based on assembly, it is known that the maximum stress located in screw than femur and plate. A region with a large strain is on the screw located in threading which closest to the screw head, with distribution color of yellow to red. This thread located at the border area between plate and femur. So we noted that stress and strain have same region that indicate maximum value. Figure 4. and Figure 5. Show the distribution for screw and other components for diameter $4.5 \mathrm{~mm}$. For all of other diameter variants of screw show the same spectrum distribution at the same region too for von Mises stress and strain also.

Of the overall variation in the diameter screw, seen the value of von Mises stress and strain becomes greater when the screw diameter gets smaller. The average size of the stress strain increase of $20-70 \%$ when the diameter gets smaller It is apparent that the screw diameter greatly affects the value of stress and strain. Meanwhile, the experimental/numerical study of Dubov et al. found screw stresses were $30 \%$ greater than the plate in the optimal fixation construct. Furthermore, in both studies, the maximum stress in the plate was observed around the screw-holes in the vicinity of the gap, and the highest stress within the femur was found around the bone screwholes [18]. The maximum stress of the screw was found around the thread also

Orthopedic screws are the most commonly used devices for bone fracture fixation. Screws are primarily responsible for retaining the stability of most screw-plate fixation devices and are commonly associated with failure due to pull-out associated with poor screw purchase or bone loss. Therefore, particular attention should be placed on the type of screws in use and their placement in bone. Primarily responsible for maintaining the stability of plate-bone constructs, and supplying necessary interfragmentary compression, screw-hold in bone is very crucial. It is well known that geometric parameters influence the pull-out strength of orthopedic screws [1921], however they effect the long-term bone-screw interaction is largely unknown. Pull-out test performed in vivo and in synthetic samples [19-22], have suggested a 
link between a screw's geometry, material properties, and its pull-out strength.

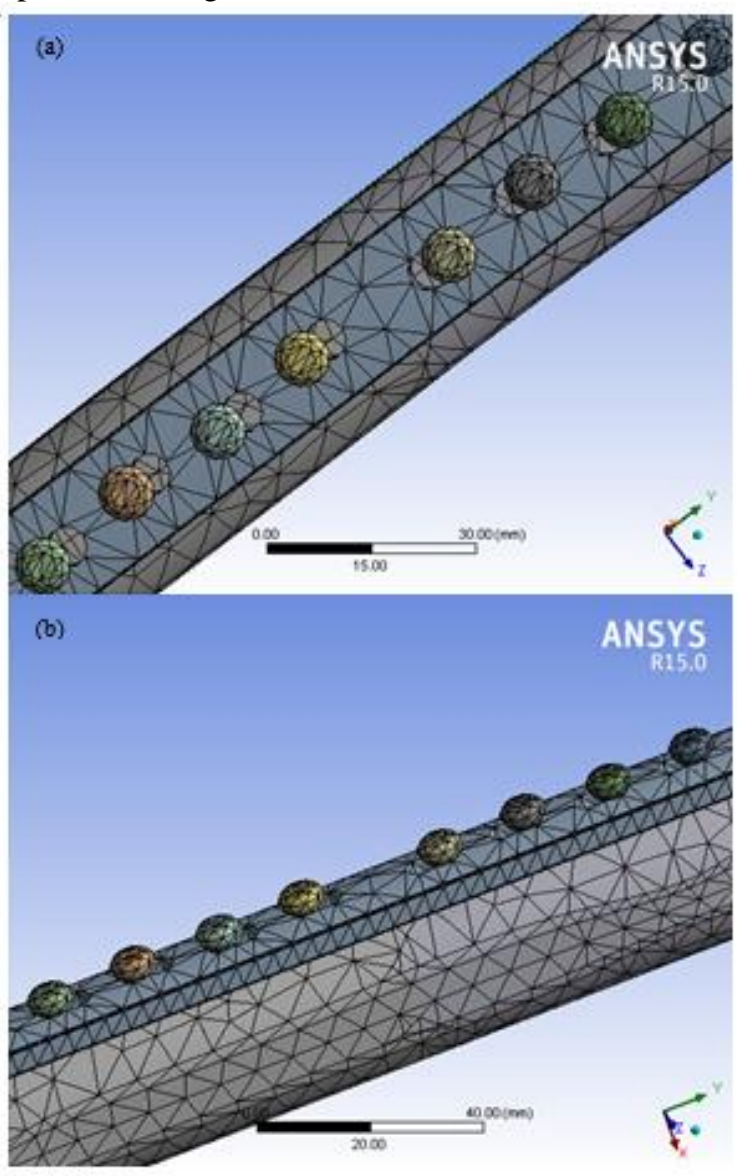

Figure 2. Assembly of all components (a) top view, and (b) side view

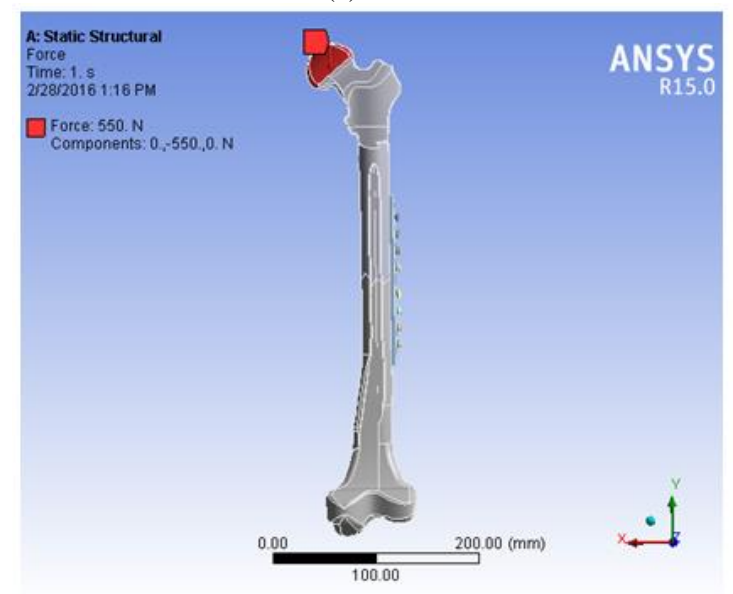

Figure 3. Loading of assembly

Figure 6. shows the distribution of total deformation spectrum. The total deformation pattern is similar to stress and strain pattern. There are shades of dark blue to red region. Dark blue region shows the minimum value of total deformation, and vice versa ie red areas have maximum value. The picture showed the spectrum of color that represents the total deformation distribution in assembly (Figure 6a.), femur (Figure 6b.), plate (Figure 6c.) and screw (Figure 6d.). Total deformation in assembly follows the direction of given load. Region near the given load had the maximum total deformation. When the region shift away from given load, then total deformation value decrease. We noted that the maximum total deformation was $1.897 \mathrm{~mm}$ located at the top of femur (at the given load point). The load distributes to region below and transferred to plate and screw. Although the maximum total deformation located in femur, we can know the total deformation in plate and screw. Total deformation in plate have same pattern with femur. The maximum value which indicated with red color located on the top plate near the given load point in femur. This value reduced as the area to the bottom plate which indicated with blue color.

Furthermore, maximum total deformation is found at the top screw near the given load point in femur. It is the different screw which has maximum von Mises stress and strain value. A region with a maximum total deformation is located at the screw head in the opposite direction of loading, marked with red color distribution. It is indicate that there are insistences of screw from plate when exposed to load. Screw head function holds plat from moving plate and detaches to femur, so that the head of the screw undergo large deformations. The thread region of screw also show gradations of color from orange to blue. The color gradations at screw body are harmony with color gradations at the screw head. Deformation value at the end of the screw head is maximum indicated with red color. But the value is very small and less than 1 $\mathrm{mm}$, very much below the deformation on femur. Because its value is very small, the shape deformation is not obvious. The direction of total deformations distribution are also in the direction of deformation on the plate and femur. In magnification, deformation occurs in the screw is in the form of increased screw curved toward the source of the load. Meanwhile, in the screw body shows gradations of color from orange to blue. The color gradation harmony with color gradations are in the screw head. A region with a maximum deformation is the end of the screw head in the opposite direction to the direction of loading, marked with red color spectrum distribution.

Of the overall variation in the diameter screw, seen the value of the average total deformation becomes great when the screw diameter gets smaller. The average size of the total deformation increase of $0.14 \mathrm{~mm}$ when the diameter gets smaller according to the results of research. It is apparent that the screw diameter greatly affects the value of total deformation. Small screw diameter is unable to sustain the bone and plate well, indicated with high value of total deformations. This further strengthens the hypothesis that for a small screw diameter will occurs plastic deformation, causing mechanical failure and can not fix the fracture. The purpose of orthopedic implants in the form of plate and screw is to fix fractures by way of pasting it back, so the conditions are ideal for both implants are not supposed to happen plastic deformation.

As expected, the resistance to bone shearing proves to be predominantly reliant upon the density of the host material [23]. However, the screw's outer diameter and length engagement critically affect the holding strength of screws in bone $[19,20]$. Other characteristics, such as screw pitch inner diameter and thread profile shape contribute to the holding power as well, but to a lesser degree [18-20]. Although sufficient pull-out strength is necessary to prevent initial screw avulsion, it is not a good indicator of potential stress shielding effects. Clinically, if pull-out of the screw is a concern because of soft bone, a larger thread diameter may be preferred, whereas if the 
bone is strong and fatigue is more of a concern, a screw with a wider root diameter will have a higher resistance to the fatigue failure.

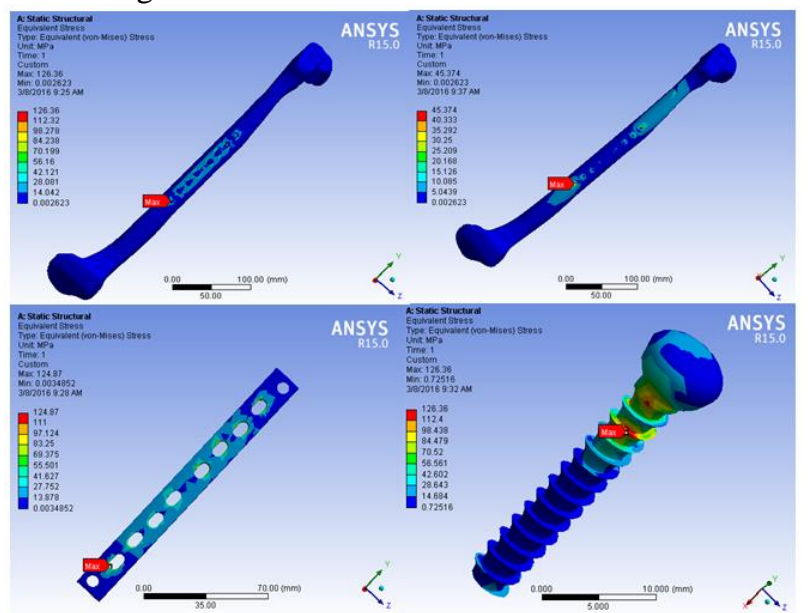

Figure 4. Von Mises stress distribution of (a) assembly, (b) femur, (c) plate, and (d) screw

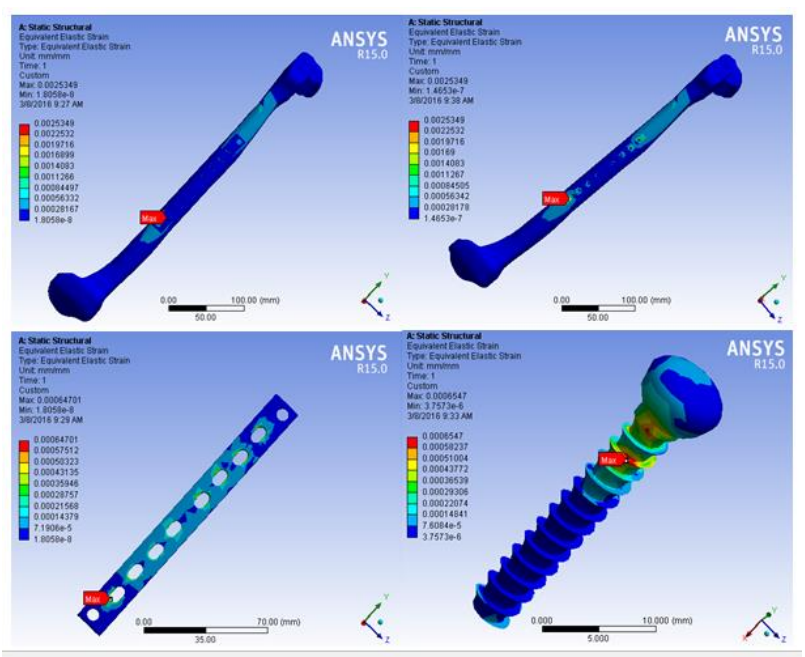

Figure 5. Strain distribution of (a) assembly, (b) femur, (c) plate, and (d) screw

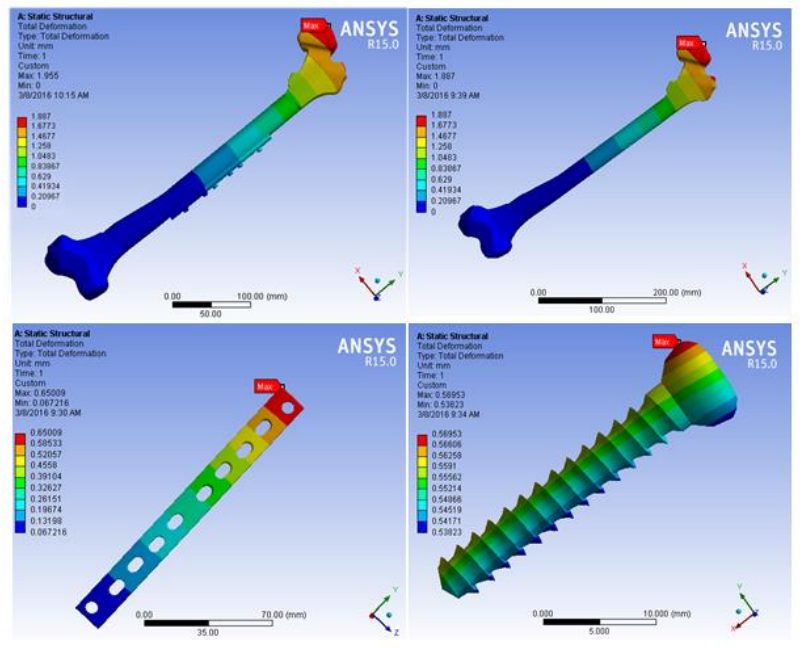

Figure 6. Total distribution of (a) assembly, (b) femur, (c) plate, and (d) screw

Since the screw remains attached to the bony tissue after it is healed, it may diminish the bone's strength and stiffness. The significantly stiffer metallic screw carries most of the shared load, causing the adjacent bone to be atrophied in response to the diminished load it is carrying, in accordance with Wolff's law of functional adaption. Wolff's law states that every change in the form and function of the bone is followed by certain definite change in their internal architecture and equally definite secondary alterations in their external conformation, in accordance with mathematical laws. The effect of metallic screws on the bony tissue in their vicinity is called stress shielding. The biomechanical compatibility of a particular screw with bone can, therefore, be characterized by the stress (or strain, or any other mechanical stimulus) distribution developing in the bone around the screw as a result of the screw's tightening during implantation [24]. Screw loosening is a common problem faced in bone fracture fixation. Stress shielding around screw threads is partially responsible for excessive bone resorption. Orthopedic screws are primarily responsible for retaining the stability of most fracture fixation devices, and are commonly associated with failure due to pull-out associated with poor screw purchase or bone loss [24]. Bone, under normal physiological conditions, is an organ of optimal design, as it maintains both mechanical and chemical homeostasis. Bone remodeling activities serve to remove bone mass where the mechanical demands of the skeleton are low, for instance in the vicinity of orthopedic implants, and form bone at those sites where mechanical loads are transmitted sufficiently [26].

Screws are primarily used to supply necessary interfragmentary compression, as standalone fixators and in conjunction with other orthopedic hardware devices, particularly plates, and so their holding power within bone is crucial. Bone, on the other hand, is a dynamic connective tissue that gives form and support to the body, while protecting vital organs, and facilitating locomotion. It also acts as a reservoir for ions, especially for calcium and phosphate, the homeostasis of which is essential to life. These functions place serious requirements on the mechanical properties of bone, which should be stiff enough to support the body's weight and tough enough to prevent easy fracturing. As well, bone must be resorbed and/or formed depending on the mechanical and biological requirements of the body [27]. Fracturefixation by bone-plate is proposed to provide immobilization at the fracture site and decrease the fracture gap, thus allowing primary bone healing or healing by endosteal callus formation. The role of the bone-plate is to hold the fractured bone segments in position, occurring at critical compressive stresses in order to accelerate healing. The complications pertaining to plate-fixation are loosening of screws under loading, local effects on vascularity of the cortex beneath the plate and extreme shielding of stresses from the bone. For these reasons, bone plates should have the appropriate biological and mechanical properties [28]. The slotted or region of the ramps at the plate, affecting the stress distribution on the screw and minimize the localized stress at one point.

\section{CONCLUSION}

This computational study has shown that the stress distribution was greatly affected by the diameter of the screws. It was found that the optimum diameter of screw was $4.5 \mathrm{~mm}$ with value of $126.36 \mathrm{MPa}$, and below the 
yield strength value of SS316L with $170 \mathrm{MPa}$. Screw must be strong and flexible to prop the broken bone, but it should not exceed the strength of the bone itself. If the value surpass the yield strength, the materials will deform plastically and broken so it is avoided. The value in this research indicates the screw's not deformed and broken so safe for femur. It is apparent that the screw diameter also greatly affects the value of total deformation. Small screw diameter is unable to sustain the bone and plate well, indicated with high value of total deformations. The maximum deformation value in screw is very small and less than $1 \mathrm{~mm}$, so the shape deformation is not obvious. This research is expected the quicker recovery time and not likely to cause post-operative pain which indicated with minimum von Mises stress value (below yield strength material) and lower deformation value.

\section{REFERENCES}

[1] D. T. Reilly and A. H. Burstein, "The Elastic and Ultimate Properties of Compact Bone Tissue," J Biomech 8, pp 384-405, 1975.

[2] B. D. Ratner, A. S. Hoffman, F. J. Schoen, and J. E. Lemons, "Biomaterials Science: An Introduction to Materials in Medicine," Vol. 2, New York Academic Press, 1996.

[3] A. Koistinen, "Improvement of The Orthopaedic Bone Screws by DLC Coatings," Publications of The University of Eastern Finland, Dissertation in Forestry and Natural Science, No 89, Nov. 2012.

[4] B. K. Kwon, D. J. Goertzen, P. J. O'Brien, H. M. Broekhuyse, and T. R. Oxland, "Biomechanical Evaluation of Proximal Humeral Fracture Fixation Supplemented with Calcium Phosphate Cement," Journal of Bone and Joint Surgery America 84, pp 951-961, 2002.

[5] C. Cuny, M. M. Scarlat, M. Irrazi, P. Beau, V. Wenger, N. Ionescu, and A. Berrichi, "The Telegraph Nail for Proximal Humeral Fractures: A Prospective Four Year Study,” Journal of Shoulder and Elbow Surgery 17, pp 539-545, 2008

[6] R. W. Gaines Jr, "The Use of Pedicle-Screw Internal Fixation For The Operative Treatment of Spinal Disorders," The Journal of Bone and Joint Surgery (American) 82, pp 1458-1476, 2000.

[7] F. A. Barber, M. A. Herbert, R. C. Beavis, and F. Barrera Oro, "Suture Anchor Materials, Eyelets, and Designs: Update 2008." Arthroscopy: The Journal of Arthroscopic and Related Surgery 24, pp 859-867, 2008

[8] K. C. Owsley and J. T. Gorczyca, "Displacement/Screw Cutout after Open Reduction and Locked Plate Fixation of Humeral Fractures," Journal of Bone and Joint Surgery America 90, pp 233-240, 2008.

[9] P. S. D. Nasr, et al., "Effect of Screw Position on Bone Tissue Differentiation Within a Fixed Femoral Fracture," Journal of Biomedical Science and Engineering, pp 71-83, 2013.

[10]M. O. Kaman, N. Celik, and S. Karakuzu, "Numerical Stress Analysis of The Plates Used to Treat The Tibia Bone Fracture," Journal of Applied Mathematics and Physics, pp 304-309, May 2014.

[11]C. H. Lee, et al., "Simulation-based Particle Swarm Optimization and Mechanical Validation of Screw Position and Number for The Fixation Stability of A Femoral Locking Compression Plate," Journal Medical Engineering and Physics, pp 57-64, 2014.
[12]B. Sepheri, E. Taheri, and R. Ganji, "Biomechanical Analysis of Diversified Screw Arrangement on 11 Holes Locking Compression Plate Considering Time-Varying Properties of Callus", Elsevier Ltd, 2013.

[13]P. S. D. Patel, D. E. T. Shepherd, and D. W. L. Hukins, "The Effect of Screw Insertition Angle and Thread Type on The Pullout Strength of Bone Screw in Normal and Osteoporotic Cancellous Bone Models," Journal of Medical Engineering and Physics, Elsevier 2010.

[14]H. K. Uhthoff, P. Poitras, and D. S. Backman, "Internal Plate Fixation of Fractures: Short History and Recent Developments," Journal of Orthopedic Science 11, pp 118-26, 2006.

[15]M. S. Ali, T.A. French, G. W. Hastings, T. Rae, N. Rushton, E. R. Ross, et al., "Carbon Fibre Composite Bone Plate. Development, Evaluation and Early Clinical Experience," Journal of Bone and Joint Surgery 72B, pp 586-91, 1990.

[16]N. Setiawan, "Peningkatan Kebutuhan Protein Hewani di Jawa Barat : Dampak dari Perubahan Struktur Penduduk", Jurnal Ilmu Ternak Vol 8 No.1, pp 65-71, 2008.

[17] A. N. Hughes and B. A. Jordan, "The Mechanical Properties of Surgical Bone Screws and Some Aspects of Insertion Practice," Journal of Injury, pp 25-38, 1972.

[18]A. Dubov, et al., "The Biomechanics of Plate Repair of Periprosthetic Femur Fractures Near The Tip of a Total Hip Implant: The Effect of Cable-Screw Position." in Proceedings of the Institution of Mechanical Engineers, pp. 857-865, 2011.

[19]S. E. Asnis, et al., "Cancellous Bone Screw Thread Design and Holding Power," Journal of Orthopaedic Trauma, pp 391-398, 1996

[20] J. R. Chapman, et al., "Factors Affecting The Pullout Strength of Cancellous Bone Screws," Journal of Biomedical EngineeringTransaction of Asme, pp 391-398, 1996.

[21]T. A. Decoster, et al., "Cancellous Bone Screw Thread Design and Holding Power," Clinical Research, pp A213-A213, 1990.

[22] J. D. Thompson, J. B. Benjamin, and J. A. Szivek, "Pullout Strengths of Cannulated and Noncannulated Cancallous Bone Screwa," Clinical Orthopaedics and Related Research, pp 241-249, 1997.

[23]R. E. Tooms, "Amputations of Lower Extremity, in Campbell's Operative Orthopaedics," A. H. Crenshaw, Editor, pp 740-760, 1992.

[24]A. Gefen, "Optimizing The Biomechanical Compatibility of Orthopedic Screws for Bone Fracture Fixation,” Journal of Medical Engineering and Physics, pp 337-347, 2002.

[25]R.M. Pilliar, et al., "Bone Ingrowth and Stress Shielding with A Porous Surface Coated Fracture Fxation Plate," J. Biomed. Mater Res 13, pp 799, 1979.

[26] G. Rouhi, "Biomechanics of Osteoporosis: The Importance of Bone Resorption and Remodeling Processes," InTech, pp 59, 2012.

[27]K. Haase, "Prediction of Stress Shielding Around An Orthopedic Screw: Using Stress and Strain Energy Density as Mechanical Stimuli," Computers in Biology and Medicine 43, pp 1748-1757, 2013.

[28] S. Benli, S. Aksoy, H. Havitcioglu, and M. Kucuk, "Evaluation of Bone Plate with Low-stiffness Material in terms of Stress Distribution," Journal of Biomkechanics 41, pp 3229-3235, 2008. 